\title{
A PSICOLOGIA COGNITIVA EXPERIMENTAL CINQÜENTA ANOS DEPOIS: A CRISE DO PARADIGMA DO PROCESSAMENTO DE INFORMAÇÃO ${ }^{1}$
}

\author{
Ederaldo José Lopes, Renata Lopes ${ }^{2}$ \\ Universidade Federal de Uberlândia \\ João de Fernandes Teixeira \\ Universidade Federal de São Carlos
}

\begin{abstract}
Resumo: Este texto apresenta uma discussão acerca da revolução cognitiva em Psicologia e de sua problemática teórico-metodológica que parece culminar numa tentativa de naturalização do conhecimento e numa dificuldade de integração da experimentação, modelagem computacional da mente e neurociências. Três abordagens alternativas/complementares ao processamento da informação são apresentadas: a abordagem computacional, as neurociências e a psicologia ecológica.
\end{abstract}

Palavras-chave: processamento da informação; método experimental; abordagem computacional; neurociências, psicologia ecológica.

\section{EXPERIMENTAL COGNITIVE PSYCHOLOGY FIFTY YEARS LATER: THE CRISIS OF THE INFORMATION-PROCESSING PARADIGM}

\begin{abstract}
This text presents a discussion about the cognitive revolution in Psychology and the questions about its theory and methodology, which appear to culminate in an attempt to make knowledge as a natural matter, and present difficulty in integrating experimentation, computer modeling of the mind and neuroscience. Three alternative/complementary approaches to information-processing are presented: computational, neurosciences and ecological psychology.
\end{abstract}

Key-words: information-processing; experimental method; computational approach; neurosciences, ecological psychology.

\section{Psicologia Cognitiva: Aspectos Históricos}

Este trabalho objetiva apresentar um quadro histórico da abordagem ou "paradigma” do processamento de informação que predominou na psicologia cognitiva em seus primórdios, mas que se encontra num estágio que necessita de revisões face ao desenvolvimento de novas abordagens, sobretudo no campo da computação e das neurociências. Sem ser exaustivo, o artigo apresenta aspectos históricos, características do paradigma do processamento de informação (PI) e algumas dessas novas abordagens que colocam desafios importantes para o PI sem, entretanto, constituírem seus subs-

Artigo recebido para publicação em 05/09/2003; aceito em 10/12/2003. ${ }^{2}$ Endereço para correspondência: Ederaldo José Lopes - Av. Pará, 1720 Campus Umuarama, Bloco 2C - Sala 2C 48, CEP.38405-320 Uberlândia, Minas Gerais, Brasil - E-mail: ederaldol@umuarama.ufu.br titutos, mas elas complementam e preenchem possíveis lacunas deixadas pelo paradigma.

A psicologia cognitiva moderna ou processamento de informação surgiu na década de 50 do século XX e pode ser considerada um novo "paradigma" no sentido kuhniano (retirando-se a exigência do critério da "universalidade de acordo"; Carone, 1998). De acordo com Mandler (1981, citado em Gardner, 1995):

Por razões que são obscuras no momento, as várias tensões e inadequações da primeira metade do século XX contribuíram para produzir um novo movimento na psicologia que primeiro adotou o rótulo de processamento de informação e depois ficou conhecido como psicologia cognitiva moderna. E tudo isto aconteceu no periodo de cin- 
co anos, entre 1955 e 1960. A ciência cognitiva surgiu durante este periodo de cinco anos, um acontecimento que está apenas começando a se tornar evidente para aqueles que a exercem.

A psicologia cognitiva moderna se insere num movimento que alguns chamam de "revolução cognitiva” (Baars, 1986; Gardner, 1995; Miller, 2003; para uma crítica da "revolução", O’Donohue, Ferguson \& Naugle, 2003). Seu surgimento coincide com a fundação de uma nova ciência da mente, interdisciplinar, composta por disciplinas tão diversas quanto a Filosofia, as Ciências da Computação, a Psicologia, da qual estamos falando. Esse surgimento concomitante da psicologia cognitiva e das ciências cognitivas fez com que a psicologia cognitiva nascesse não meramente como um novo sistema psicológico, com objetivos bastante diferentes do behaviorismo praticado até aquele momento. Tratava-se de uma nova abordagem que dava continuidade ao uso do método experimental, tal qual seu antecessor, mas ao mesmo tempo estava inserido num contexto interdisciplinar "oficial”, marcado por importantes encontros de cientistas renomados da época, tais como o simpósio Hixon de 1948 no Instituto de Tecnologia da Califórnia e os vários encontros realizados em 1956 no Instituto de Tecnologia de Massachusetts. Entre os fatores precipitantes desse movimento interdisciplinar, podemos citar os seguintes:

a) a invenção do computador (Crane, 1995; Eysenck \& Keane, 1994; French \& Colman, 1995; Thagard, 1998), de onde nasceu a idéia central do cognitivismo, ou seja, de que os processos mentais seriam processos computacionais e simbólicos;

b) a neuropsicologia, a partir dos estudos de pacientes com lesões cerebrais, especialmente provenientes das tragédias da II Guerra Mundial. Esses estudos forneceram importantes "insights" sobre o funcionamento cerebral de pessoas normais;

c) a teoria matemática da informação (Shannon \& Weaver, 1949), a partir da qual se desenvolveu a idéia de que a informação poderia ser concebida de tal forma a ser totalmente desvencilhada de qualquer conteúdo específico, mas ela era, antes de tudo, uma decisão única realizada entre duas alternativas possíveis;

d) a cibernética, que trouxe importantes descobertas a respeito dos mecanismos de feedback e dos sistemas autocorretores e auto-reguladores.

e) a retomada do tempo de reação (TR) na medida dos processos mentais (Galera \& Lopes, 1995), com o uso conseqüente dos processos inferenciais do funcionamento mental;

f) mudanças na concepção positivista de ciência até então aceita, que consistiram, sobretudo, na crítica da noção de objetividade científica, no surgimento da noção de revolução científica e paradigma (Eysenck \& Keane, 1994; Lachman, Lachman \& Butterfield, 1979; Masterman, 1979; Popper, 1979).

\section{Características da Abordagem do Processamento de Informação}

Palmer e Kimchi (1986) apresentaram uma visão extensiva sobre as características da abordagem do PI dentro da psicologia. Isto porque, segundo eles, nos fundamentos dessa abordagem se encontram filósofos e cientistas da computação. Dos primeiros teriam vindo formulações que culminaram no que se chama de funcionalismo, uma linha de investigação em filosofia da mente segundo a qual a mente ou os estados mentais são caracterizados pelo papel funcional que exercem no sistema, independentemente do tipo de substrato físico (Putnam, 1997; Teixeira, 2000). Dos últimos advieram propósitos relacionados à simulação dos processos mentais nos computadores digitais (Newell, 1980; Newell \& Simon, 1972; Pylyshyn, 1984; Ross, 1997). Para muitos psicólogos cognitivos da abordagem do PI, algumas suposições apresentadas pelas duas visões anteriores podem parecer muito fortes ou erradas e não refletem a natureza da abordagem PI na psicologia. Assim, a fim de caracterizar mais claramente essa abordagem dentro da psicologia, Palmer e Kimchi (1986) apresentaram 5 suposições sobre o PI:

1. Descrição informacional: os eventos mentais podem ser descritos como eventos informacionais e consistem de três partes: entrada da informação, operações sobre a informação e a saída. Por eventos mentais, eles querem dizer todos os eventos consci- 
entes, bem como todos os acontecimentos que influenciam o comportamento. Psicologicamente falando, nesse nível é importante notar que interessa aos psicólogos a noção do conteúdo representacional mais que a noção de informação como uma quantidade mensurável conforme prevista na formalização de Shannon. A visão psicológica da informação é como um evento abstrato, uma descrição teórica de eventos mentais e não pela qualidade de surpresa ou incerteza contida na mensagem, medida matematicamente. Isso quer dizer que dois sinais muito diferentes fisicamente podem estar transmitindo a mesma informação, com o mesmo significado. Discutida nesse nível, o PI fornece a possibilidade de mapear as informações de entrada e as informações de saída. Muito embora as teorias de mapeamento não forneçam como os mapeamentos input-output são realizados, elas fornecem conhecimentos que permitem restringir possíveis mecanismos que não são plausíveis, permitindo o teste da teoria, uma vez que essas teorias lidam diretamente com a realidade empírica, podendo especificar quando o input e output terminam de modo a compará-los com o comportamento humano.

2. Decomposição recursiva: qualquer evento informacional unitário pode ser descrito de forma mais analítica, através da decomposição dele em eventos mais simples. Cada um desses eventos também é um evento informacional, e as relações de ordem temporal entre eles especifica como a informação flui através do sistema de componentes. As teorias informacionais que fazem uso dessa suposição são verdadeiras teorias de PI (modelos de processos cognitivos), porque elas entram na "caixa preta”. É o que Marr (1982) chama de nível algorítmico da teoria. Esse nível, portanto, especifica as operações mentais e aparecem representadas na literatura da psicologia cognitiva em forma de diagramas de fluxo da informação. A decomposição significa que uma operação na memória, por exemplo, possa ser decomposta em vários níveis e representada por diagramas. A natureza recursiva da decomposição significa a construção de uma hierarquia das operações realizadas numa dimensão vertical (operações de alto nível $\mathrm{x}$ de baixo nível ou descrições gerais $\mathrm{x}$ específicas) e numa dimensão horizontal que simplesmente enumera os componentes que pertencem a cada nível do diagrama de fluxo da informação.

3. Continuidade de fluxo: toda a informação necessária para realizar cada operação deve estar disponível na saída das operações e formam a base de entrada da próxima informação, representada pelos "boxes” do diagrama. Ela é um corolário da suposição da decomposição.

4. Dinâmica de fluxo: Nenhuma informação de saída pode ser produzida por uma operação até que sua informação de entrada esteja disponível para realizá-la e tenha passado tempo suficiente para que a entrada seja processada. A dinâmica do fluxo da informação é importante para o teste empírico da teoria. As restrições são que o processamento não pode começar até que alguma informação de entrada esteja disponível e que toda operação toma alguma quantidade de tempo. Uma suposição adicional é que cada operação ao longo do fluxo constitui um estágio de processamento discreto, suposição amplamente testada a partir do trabalho de "memory scanning” proposto por Sternberg $(1966,1969)$ e que gerou inúmeros trabalhos subseqüentes (Lopes, 1992; Sternberg, 1975; 2001). Outras concepções da dinâmica de fluxo também foram desenvolvidas. Por exemplo, Normam e Bobrow (1975) propuseram uma dinâmica de fluxo mais flexível que a anterior, sugerindo uma "saída disponível continuamente", mais tarde desenvolvida matematicamente por McClelland (1979) e Miller (1988), ficando conhecida como "processamento em cascata". Neste tipo de processamento, cada operação começa produzir alguma saída assim que surge alguma entrada, de modo que os estágios estão sempre sendo indexados pela presença das entradas. Não há uma decisão final sobre qual modelo de PI é mais plausível. O importante são as condições amplas de testagem desses modelos, o que lhes confere um poder científico maior dentro da tradição de falseamento das teorias (Popper, 1959/1999).

5. Incorporação física: no sistema físico dinâmico cujo comportamento está sendo descrito como um evento informacional, a informação é realizada pelos estados do sistema (chamadas de representação), e as operações são realizadas pelas mudanças de estado (chamadas de processos) (sobre informação e representação mental, Dietrich \& Markman, 2003; Markman \& Dietrich, 2000 a; 2000b). 
Um outro conjunto de características do PI, similar ao de Palmer e Kimchi (1986), porém mais resumido, foi proposto por Eysenck e Keane (1994):

(I) As pessoas são consideradas seres autônomos e intencionais que interagem com o mundo externo;

(II) a mente através da qual elas interagem com o mundo é um sistema de processamento de símbolos de uso geral;

(III) nos símbolos atuam vários processos, que os manipulam e os transformam em outros símbolos que, por fim, terão relação com as coisas do mundo externo;

(IV) a meta da pesquisa psicológica é a de especificar os processos simbólicos e representações subjacentes ao desempenho de todas as tarefas cognitivas;

(V) os processos cognitivos levam tempo; sendo assim, suposições sobre os tempos de reação podem ser feitas ao se presumir que certos processos ocorrem em seqüência e/ou possuem alguma complexidade especificável;

(VI) a mente é um processador de capacidade limitada, que tem limitações tanto estruturais quanto de recursos;

(VII) o sistema simbólico depende de um substrato neurológico, mas não está inteiramente limitado por este.”

Muito embora o PI não tenha fornecido uma teoria abrangente da cognição humana (uma arquitetura cognitiva), ele tem servido como um paradigma (ou arcabouço), ou seja, um conjunto maleável de idéias que podem ser utilizadas na formulação de teorias cognitivas.

Dessas características, é preciso salientar três aspectos importantes em termos das discussões futuras: primeiro, a continuidade da influência do modelo computacional da mente, surgida com os primeiros modelos computacionais de processamento de informação (SGP: Solucionador Geral de Problemas, Newell, Shaw \& Simon, 1958); segundo, a continuidade da experimentação em psicologia cognitiva, reavivada com o emprego do tempo de reação (TR) para a medida de processos mentais, a partir da metodologia apresentada por Donders (1868/1969). Mencione-se o fato de que à época da "revolução cognitiva”, esses estudos começaram com a investigação das relações entre incerteza e tempo de reação (Hick, 1952; Hyman, 1953) e mais tarde tiveram um enorme impacto nos estudos de decomposição do TR em estágios de processamento (Sternberg, 1966, 1969, 2001); terceiro, a afirmação do substrato neural para o processamento de informação, um elemento importante que adquiriu maior relevo a partir de 1990 (Lopes, Lopes \& Rodrigues, 2000; Wagemans, Verstraten \& He, 2001) e que vem influenciando renomados psicólogos cognitivos a fazerem neurociência cognitiva há algum tempo, como é o caso de Michael Posner \& Cols. (Posner, 1992; Posner \& Dehaene, 1994; Posner \& Levitin, 1999; Posner \& Petersen, 1990; Posner, Petersen, Fox \& Raichle, 1988). Acrescente-se um quarto elemento - crítico dos modelos de PI - que é quanto ao papel exercido pelo contexto cultural na constituição da mente.

Essas tendências teórico-metodológicas dão o direcionamento e os desafios futuros da psicologia cognitiva em relação às ciências cognitivas. A abordagem do PI não isola a psicologia cognitiva das ciências cognitivas. Pelo contrário, a base do PI é a noção de mente como um conjunto de computações que ocorrem num substrato neural. Além disso, dessa relação interdisciplinar se espera a reunião de condições para a construção de modelos e teorias psicológicas mais poderosas e de maior cientificidade.

\section{A Crise do Processamento de Informação e Algumas Alternativas}

As ciências cognitivas têm servido, por um lado, para fazer descobertas a respeito do funcionamento da mente, mas por outro têm dado margem a questionamentos sobre a continuidade da existência da filosofia (Oliveira, 1992; Gardner, 1995). Assim como a filosofia corre o perigo de ser substituída pelas ciências cognitivas, a psicologia cognitiva experimental (Processamento de Informação) também tem tido sérias dificuldades em se consolidar como um sistema psicológico forte, substituto do behaviorismo, pois ao mesmo tempo em que se propôs a ser uma ciência da mente (ou parte dela), continuou a fazer da experimentação seu método de investigação privilegiado. O uso exagerado, quase exclusivo, do método experimental pode ter se confi- 
gurado numa amarra incapaz de dar conta da vastidão dos fenômenos cognitivos. Ademais, o compromisso interdisciplinar dentro das ciências cognitivas também não se consolidou, de modo que as descobertas das neurociências ou da computação precisam ser integradas em modelos mais amplos de funcionamento cognitivo. Ocorreu, então, um certo isolamento da psicologia cognitiva em relação às demais áreas das ciências cognitivas, de modo que, fora da literatura da psicologia, os manuais ou mesmo os periódicos falam da grande área, as ciências cognitivas, muitas vezes sem se referir à psicologia cognitiva. Então, nós nos perguntamos: Qual é o futuro da psicologia cognitiva experimental, que buscou fundamentos para os pressupostos do processamento da informação?

A crise do paradigma não se resume aos limites impostos pela experimentação. A experimentação fornece dados que sustentam teorias/modelos dentro da psicologia. Mas a vida mental é muito mais do que o captado em situações artificiais, de modo que o uso indiscriminado do método experimental tem sido a base de um movimento circular de teste de teorias dentro da psicologia cognitiva (Neisser, 1997). As conclusões advindas de outras áreas certamente são importantes e, em certa medida, podem desafiar conclusões obtidas via experimentação, mas consideramos esse movimento salutar para o desvendamento do conhecimento das funções mentais. Assim, podemos apresentar resumidamente três movimentos alternativos, que podem ser vistos também como complementares àquelas noções tradicionais do processamento da informação: a abordagem computacional, as neurociências e a psicologia ecológica.

1) A Abordagem Computacional: Nas simulações computadorizadas dos processos cognitivos os pesquisadores programam uma máquina no sentido de imitar uma dada função ou processo cognitivo humano (manipulação de objetos, imagens, reconhecimento de padrões visuais, etc). Arquiteturas inteiras têm sido propostas como forma de se entender o funcionamento mental, e muita discussão tem sido produzida ao longo desses últimos anos sobre os limites e avanços dessa abordagem em termos de se compreender os processos cognitivos humanos (Garnham, 1995; Pylyshyn, 1984; Teixeira,
2000). Uma das formas de abordagem computacional da mente que surgiu em meados de 1980 e continua até hoje é o conexionismo ou redes neurais (Rumelhart e McLelland, 1986).

Muito embora algumas pessoas confundam a modelagem computacional dos processos cognitivos com a inteligência artificial, há uma diferença entre ambas: a inteligência artificial se preocupa essencialmente em criar sistemas que processem a informação de maneira eficiente e inteligente, sem querer, com isso, que esses sistemas imitem alguma função cognitiva similar ao homem, o que é objetivo da modelagem computacional da mente.

De um ponto de vista interdisciplinar, uma das vantagens da modelagem computacional é sua capacidade de ser um instrumento utilizado para se testar teorias psicológicas e fornecer elementos para o aprimoramento dos modelos e teorias cognitivas construídas e sustentadas por dados experimentais.

De acordo com Crowter-Heyck (1999), a abordagem computacional adquiriu o poder de remodelar a psicologia experimental por três razões: primeiro porque ela forneceu uma base mais sólida (ciência da computação e engenharia elétrica) para a psicologia cognitiva experimental sem reduzi-la a uma ciência básica; segundo porque colocou a psicologia experimental em contato com uma das ferramentas mais potentes do século XX (o computador) e terceiro porque ela permitiu aos psicólogos operacionalizarem muitos termos mentalistas.

Embora a abordagem computacional tenha trazido contribuições à psicologia cognitiva, a possibilidade de fusão da psicologia cognitiva/abordagem computacional está na agenda de alguns. Nessa direção, Gardner (1995, p. 152) afirma que:

a fusão da psicologia cognitiva com a inteligência artificial ocupará a região central de uma nova ciência cognitiva unificada, com campos como a lingüistica, a teoria musical ou a análise espacial fornecendo as estruturas adequadas para problemas que se encontram dentro de seus domínios.

2) As neurociências: Na década de 90 (a década do cérebro), a psicologia cognitiva sofreu uma forte infusão das neurociências. Pode-se dizer que, 
paralelamente ao desenvolvimento da "Revolução Cognitiva”, uma nova revolução estava sendo traçada dentro da biologia, constituindo o que Squire e Kandel (2003) chamam de uma "Revolução Biológica”. O conhecimento sobre o cérebro no plano celular e intercelular, aliado a um conjunto de técnicas de imageamento cerebral (Imbert, 1998; Kandel, Schwartz, \& Jessell, 1997), têm permitido se pensar na eliminação da psicologia "folk" em detrimento de um processo de naturalização da mente, reduzindo a psicologia às neurociências (Searle, 1997; Teixeira, 2000). Para Hatfield (2002)

A few recent thinkers believe that psychology can remain scientific only by becoming something else: neuroscience, cognitive science, or those and more (p. 207).

Embora as neurociências nos ofereçam um cenário potencialmente reducionista para a psicologia, elas parecem constituir uma ferramenta imprescindível do ponto de vista teórico e prático, isto porque o conhecimento do cérebro pode trazer inúmeros benefícios, tanto no campo da psicoterapia quanto no campo da neuropsicologia. Além disso, as neurociências podem fornecer o "gap” explanatório entre a mente (o cérebro) e o comportamento, um fato reconhecido até mesmo por Skinner (1989). Para Teixeira (no prelo, p. 9), “a neurociência cognitiva abre uma perspectiva ampla de investigação que reintegra o papel do comportamento no estudo da cognição e da natureza das experiências conscientes.

3) A psicologia ecológica e validade ecológica: Ulric Neisser, um dos pais da psicologia cognitiva moderna, se rebelou fortemente contra a experimentação em psicologia cognitiva, porque esta se revela sem validade ecológica e indiferente à cultura. Primeiro a publicar um livro de psicologia cognitiva com esse rótulo (Cognitive Psychology, 1967), num tom pessimista, ele rebateu a própria obra em favor de uma psicologia ecologicamente válida (Neisser, 1976). Para ele os vilões do pedaço são os modelos mecanicistas do processamento de informação, que tratam a mente como um dispositivo de capacidade fixa para converter inputs discretos $e$ sem sentido em perceptos (percepts) conscientes.
Associada à idéia de uma psicologia ecologicamente válida, está um dos pontos mais críticos à psicologia cognitiva experimental: que ela não tem validade ecológica; ela não dá respostas para os problemas cotidianos. Esse tipo de crítica tem vários desdobramentos, um dos quais é o movimento de discussão que coloca a necessidade de se entender a mente humana de uma perspectiva menos individualista, mais calcada em aspectos evolucionários e culturais (Erneling, 1997 a, b; Neisser, 1976; 1982; 1997; Reed, 1997; Sperber \& Hirschfeld, 2001; Tooby \& Cosmides, 2002). A psicologia ecológica enfatiza as relações do homem (a mente) com o seu ambiente (ecológica), de modo que não há por que separar uma mente (individual) de seu contexto sócio-cultural, pois isto eliminaria o caráter construtivo e não-passivo do homem frente ao seu mundo e a si mesmo. Nesse sentido, os teóricos a partir de Neisser (Neisser, 1976, 1978, 1982) argumentaram sobre a real validade dos estudos experimentais e da possibilidade de se extrapolar as descobertas sobre o funcionamento da memória em ambientes artificiais (laboratórios) para a vida prática. Embora a psicologia ecológica seja um movimento forte no estudo da cognição, nos estudos sobre a memória humana, por exemplo, um intenso debate foi gerado pela postura de alguns autores que questionaram a validade dos estudos feitos em contextos naturais, pelo fato de se induzir a uma perda de controle e de generalização dos resultados, elementos necessários para se construir uma teoria válida sobre a memória (Banaji \& Crowder, 1989; Roediger, 1991). Nosso posicionamento tem sido mais de reconciliação no sentido exposto por (Baddeley 1990; Cohen, 1996), ou seja, tanto a pesquisa natural quanto experimental tem contribuído positivamente para o aumento do conhecimento sobre a memória. Não há método ótimo por si mesmo. É preciso verificar as condições e os objetivos da pesquisa e só a partir daí decidir pelo método apropriado (Capaldi \& Proctor, 2000; Kuhn, 1978; Laudan, 1977). Em outras palavras, não é o método que guia um problema de pesquisa a ser investigado, mas o mais importante é formular um bom problema de pesquisa e buscar o método mais apropriado de investigação. 


\section{Conclusões}

Conclui-se que a psicologia cognitiva experimental (processamento da informação), imersa numa verdadeira dispersão paradigmática, ainda não construiu um arcabouço a partir dos dados obtidos via método experimental ou qualquer outro método, revelando que a "universalidade de acordo” será um difícil critério científico ao qual a psicologia e a psicologia cognitiva se adequarão. Ademais, a interdisciplinaridade, apregoada durante a "revolução cognitiva”, é uma figura de retórica cujas conseqüências mais claras têm sido colocar a psicologia cognitiva num plano secundário. Pode-se perguntar, por conseguinte, qual é o papel da psicologia cognitiva dentro das ciências cognitivas? Qual é o papel real dela no processo de consolidação da "revolução cognitiva”? (Johnson \& Erneling, 1997).

Ao que tudo indica, as abordagens alternativas fornecerão elementos necessários para se compreender a cognição não só através de uma metodologia diferente, mas sobretudo buscando compreender o papel do ambiente na construção do comportamento. A compreensão da cognição não se encerra com o conhecimento computacional, cerebral ou da psicologia cognitiva experimental. Ele se ampliará na medida em que esses conhecimentos avançarem e se integrarem. Isso não significa que o futuro da psicologia cognitiva experimental seja sua naturalização, matematização ou, simplesmente, sua absorção pelas ciências cognitivas (Solso, 1999). Há muito o que se construir com a experimentação, mas há muito o que se construir com o conhecimento do ambiente, da cultura e do comportamento, inclusive nas situações clínicas. Para finalizar, citemos o pensamento psicológico de vanguarda de Alan Baddeley (1989, p. 2), na defesa da psicologia cognitiva experimental:

Os métodos computacionais têm desempenhado e continuam a desempenhar um papel central na ciência cognitiva, mas eles necessitam ser testados e avaliados por regras obtidas pela evidência empírica, o domínio da psicologia cognitiva.

\section{Referências Bibliográficas}

Baars, B.J. (1986). The cognitive revolution in psychology. New York: Guilford Press.

Baddeley, A. D. (1989). Cognitive psychology and cognitive science. Em A.D. Baddeley \& N. O . Bernsen.( Orgs.), Research Directions in Cognitive Science: A European Perspective, Vol. 1: Cognitive Psychology. London: Lawrence Erlbaum Associates, pp. 1-8.

Baddeley, A . (1990). Human memory: Theory and practice. Hove: Lawrence Erlbaum Associates.

Banaji, M.R. \& Crowder, R.G. (1989). The bankruptcy of everyday memory. American Psychologist, 44, 1185-1193.

Capaldi, E.J. \& Proctor, R.W. (2000). Laudan's normative naturalism: A useful philosophy of science for psychology. American Journal of Psychology, 113(3), 430-454.

Carone, I. (1998). A questão dos paradigmas em psicologia - um balanço crítico. Psicologia em Estudo, 3(1), 191-200.

Cohen, G. (1996). Memory in the real world. Hove: Psychology Press.

Crane, T. (1995). The mechanical mind: a philosophical introduction to minds, machines and mental representation. London: Penguin Books.

Crowther-Heyck, H. (1999). George A. Miller, language, and the computer metaphor of mind. History of Psychology, 2(1), 37-64.

Dietrich, E. \& Markman, A . B. (2003). Discrete thoughts: Why cognition must use discrete representations. Mind \& Language, 18, 95-119.

Donders, F.C. (1868/1969). On the speed of mental processes. (Transl. By W.G. Koster) Acta Psychologica, 30, 412-431.

Erneling, C. (1997 a). Challenges to cognitive science: The cultural approach. Em D.M. Johnson \& C. E. Erneling (Orgs.). The future of the cognitive revolution (pp. 275-278). New York: Oxford University Press.

Erneling, C. (1997b). Cognitive science and the 
future of psychology: Challenges and opportunities. Em D.M. Johnson \& C. E. Erneling (Orgs.). The future of the cognitive revolution (pp. 376-382). New York: Oxford University Press.

Eysenck, M.W., \& Keane, M.T. (1994). Psicologia cognitiva: Um manual introdutório. Porto Alegre: Artes Médicas.

French, C.C., \& Colman, A . M. (1995). Cognitive psychology. New York: Longman Pubs.

Galera, C., \& Lopes, E.J. (1995). Cronometria de processos mentais. Temas em Psicologia, 3, 1-10.

Gardner, H. (1995). A nova ciência da mente: uma história da revolução cognitiva. São Paulo: Edusp.

Garnham, A. (1995). Artificial intelligence. Em C.C. French \& A.M. Colman (Orgs.). Cognitive psychology. New York: Longman Publ.

Hatfield, G. (2002). Psychology, philosophy, and cognitive science: Reflections on the history and philosophy of experimental psychology. Mind \& Language, 17, 207-232.

Hick, W.E. (1952). On the rate of gain of information. Quarterly Journal of Experimental Psychology, 4, 11-26.

Hyman, R. (1953). Stimulus information as a determinant of reaction time. Journal of Experimental Psychology, 45, 188-196.

Imbert, M. (1998) Neurociências e ciências cognitivas. Em D. Andler (Org.) Introdução às ciências cognitivas (pp. 55-75). São Leopoldo: Unisinos.

Johnson, D.M. \& Erneling, C.E. (Orgs.) (1997). The future of the cognitive revolution. New York: Oxford University Press.

Kandel, E.R., Schwartz, J.H., \& Jessell, T.M. (1997). Fundamentos da neurociência e do comportamento. Rio de Janeiro: Prentice-Hall do Brasil.

Kuhn, T.S. (1978). A estrutura das revoluções cientificas (2a. Ed.). São Paulo: Perspectiva.

Lachman, R., Lachman, J.L. \& Butterfield, E.C.
(1979). Cognitive psychology and information processing: An introduction. Hillsdale: Lawrence Erlbaum Associates.

Laudan, L. (1977). Progress and its problems. California: University of California Press.

Lopes, E.J. (1992). Codificação de sinais visuais na memória ativa evidenciada pela aplicação do método dos fatores aditivos. Dissertação de Mestrado, Universidade de São Paulo, Ribeirão Preto, SP.

Lopes, E. J., Lopes, R. F.F. \& Rodrigues, C.M.L. (2000). Perspectivas do estudo da mente: da psicologia cognitiva às neurociências. Revista da Sociedade de Psicologia do Triângulo Mineiro, 3, 6-11.

Markman, A . B. \& Dietrich, E. (2000a). Extending the classical view of representation. Trends in Cognitive Sciences, 4, 470-475.

Markman, A . B. \& Dietrich, E. (2000b). Em defense of representation. Cognitive Psychology, 40, 138-171.

Marr, D. (1982). Vision: A computational investigation into the human representation and processing of visual information. San Francisco: W.H. Freeman.

Masterman, M. (1979). A natureza de um paradigma. Em I. Lakatos \& A. Musgrave (Orgs.), A crítica e o desenvolvimento do conhecimento. São Paulo: Cultrix/Edusp.

McClelland, J.L. (1979). On the time relations of mental processes. An examination of processes in cascade. Psychological Review, 86, 287-330.

Miller, J. (1988). Discrete and continuous models of human information processing: Theoretical distinctions and empirical results. Acta Psychologica. 67, 191-257.

Miller, G. A . (2003). The cognitive revolution: a historical perspective. Trends in Cognitive Sciences, 7, 141-144.

Neisser, U. (1967). Cognitive psychology. New York: Appleton-Century-Crofts.

Neisser, U. (1976). Cognition and Reality. San Fran- 
cisco: W. H. Freeman.

Neisser, U. (1978). Memory: What are the important questions? Em M.M. Gruneberg, P. E. Morris \& R.N. Sykes (Orgs.), Practical aspects of memory.(pp. 167-178). London: Academic Press.

Neisser, U. (1982). Memory observed. San Francisco: Freeman.

Neisser, U. (1997). The future of cognitive science: An ecological analysis. Em D.M. Johnson \& C. E. Erneling (Orgs.). The future of the cognitive revolution (pp. 247-260). New York: Oxford University Press.

Newell, A . (1980). Physical symbol systems. Cognitive science, 4, 135-183.

Newell, A ., Shaw, J.C. \& Simon, H. A . (1958). Elements of a theory of human problem solving. Psychological Review, 65, 151-166.

Newell, A. \& Simon, H. A. (1972). Human problem solving. Englewood Cliffs: Prentice Hall.

Norman, D.A. \& Bobrow, D.G. (1975). On datalimited and resource-limited processes. Cognitive Psychology, 7, 44-64.

O’Donohue, W., Ferguson, k.E. \& Naugle, A . E. (2003). The structure of the cognitive revolution: an examination from the philosophy of science. The Behavior Analyst, 26, 85-110.

Oliveira, M.B. (1992). Filosofia e ciência cognitiva. Manuscrito, XV(2), 35-63.

Palmer, S.E. \& Kimchi, R. (1986). The information processing approach to cognition. Em T. Knapp \& L.C. Robertson (Orgs.), Approaches to cognition: Contrasts and controversies. Hillsdale: Lawrence Erlbaum.

Popper, K. (1959/1999). A lógica da pesquisa científica. São Paulo: Cultrix.

Popper, K. (1979). A ciência normal e seus perigos. Em I. Lakatos \& A . Musgrave (Orgs.). A critica e o desenvolvimento do conhecimento. São Paulo: Cultrix e Edusp.

Posner, M.I. (1992). Attention as a cognitive and neural system. Current Directions in Psychological Science, 1, 11-14.

Posner, M.I. \& Dehaene, S. (1994). Attentional networks. Trends in neurosciences, 17, 75-79.

Posner, M.I. \& Levitin, D.J. (1999). Imaging the future. Em R.L. Solso (Org.), Mind and brain sciences in the 21 st. Century. Cambridge: MIT Press

Posner, M.I. \& Petersen, S.E. (1990). The attention system of the human brain. Annual Review of Neuroscience, 13, 25-42.

Posner, M.I., Petersen, S. E., Fox, P.T., \& Raichle, M.E. (1988). Localization of cognitive operations in the human brain. Science, 240, 1627-1631.

Putnam, H. (1997). Functionalism: Cognitive science or science fiction? Em D.M. Johnson \& C. E. Erneling (Orgs.). The future of the cognitive revolution (pp. 32-44). New York: Oxford University Press.

Pylyshyn, Z. (1984). Computation and cognition: Toward a foundation for cognitive Science. Cambridge: MIT Press.

Reed, E. (1997). The cognitive revolution from an ecological point of view. Em D.M. Johnson \& C. E. Erneling (Orgs.). The future of the cognitive revolution (pp. 261-274). New York: Oxford University Press.

Roediger, H.L. (1991). They read an article? A commentary on the everyday memory controversy. American Psychologist, 46, 37-40.

Ross, D.(1997). Is cognitive science a discipline? Em D.M. Johnson \& C. E. Erneling (Orgs.). The future of the cognitive revolution (pp. 102-108). New York: Oxford University Press.

Rumelhart, D. \& McClelland, J.L. \& PDP Res. Group (Orgs.) (1986). Parallel distributed processing: vol. 1, Foundations. Cambridge: MIT Press.

Searle, J. (1997). A redescoberta da mente. São Paulo: Martins Fontes.

Shannon, C.E. \& Weaver, W. (1949). The mathematical theory of communication. Urbana: University of Illinois Press. 
Skinner, B.F. (1989). The origins of cognitive thought. American Psychologist, 44, 13-18.

Solso, R.L. (1999). Mind and brain sciences in the 21st century. Cambridge: MIT Press.

Sperber, D. \& Hirschfeld, L. (2001). Culture, cognition, and evolution. Em R.A. Wilson \& F.C. Keil (Ogss.). The MIT encyclopedia of the cognitive sciences. Cambridge: MIT Press.

Squire, L.R. \& Kandel, E.R. (2003). Memória: Da mente às moléculas. Porto Alegre: Artmed.

Sternberg, S. (1966). High-speed scanning in human memory. Science, 153, 652-654.

Sternberg, S. (1969). The discovery of processing stages: extensions of Donders' method. Acta Psychologica, 30, 276-315.

Sternberg, S. (1975). Memory scanning: New findings and current controversies. Quarterly Journal of Experimental Psychology, 27, 1-32.

Sternberg, S. (2001). Separate modifiability, mental modules, and the use of pure and composite measures to reveal them. Acta Psychologica, 106, 147-246.

Teixeira, J.F. (2000). Mente, cérebro e cognição. Petrópolis: Vozes.

Teixeira, J.F. (no prelo). Behaviorismo radical e ciência cognitiva: Retomando um diálogo necessário. Arquivos Brasileiros de Psicologia.

Thagard, P. (1998). Mente: Introdução à ciência cognitiva. Porto Alegre: ArtMed.

Tooby, J. \& Cosmides, L. (2002). Toward mapping the evolved functional organization of mind and brain. Em D.J. Levitin (Org.), Foundations of cognitive psychology: Core readings. Cambridge: MIT Press.

Wagemans, J., Verstraten, F. A . J. \& He, S. (2001). Editorial: Beyond the decade of the brain: towards a functional neuroanatomy of the mind. Acta Psychologica, 107, 1-7.

\section{Agradecimento}

Parte deste artigo foi baseado no trabalho de Pós-Doutorado do primeiro autor sob a supervisão do terceiro na Universidade Federal de São Carlos, SP. Agradecimentos são devidos à Faculdade de Psicologia da Universidade Federal de Uberlândia, ao Departamento de Filosofia da Universidade Federal de São Carlos e ao Conselho Nacional de Desenvolvimento Científico e Tecnológico (CNPq-Processo No. 150059/ 03-5) pelo apoio recebido. 